\title{
Fractional Order Sliding Mode Speed Control of Feedback Linearized Induction Motor
}

Research Article

\author{
Yeshiwas Fetene ${ }^{1, *}$, Dereje Shibeshi \\ ${ }^{1}$ Electrical and computer Engineering Department, Debre Berhan University, Debre Berhan, Postal Box 445, Ethiopia \\ Ethiopian Aviation Academy, Ethiopian AirLines, Addis Ababa, Ethiopia
}

Received July 29, 2020; Accepted September 18, 2020

\begin{abstract}
Induction machines have of late become the most popular workhorses in the industry replacing DC machines because of their advantages, such as reduced cost, reliability and the absence of commutators, which make them adapt to unfavourable conditions with lower maintenance requirement. However, higher-order models of $\mathrm{AC}$ machines, nonlinearities in model equations, uncertainties in parameters and load disturbances make induction motors difficult to control than that of DC motors. In this paper, a robust control strategy considering the recent advances in technology is proposed employing input-output feedback linearization for the exact decoupling of electromagnetic torque and rotor flux using fractional-order sliding mode controller (SMC) for the outer speed loop. The sliding mode observer is also designed to extract the rotor flux from the DC input voltage of the inverter and stator current measurements. Finally, a comparison between the commonly used proportional integral and derivative (PID) controller and the proposed fractional-order SMCs is made and the conclusion is reached.
\end{abstract}

Keywords: induction motor • input-output feedback • linearization • fractional-order sliding mode controller • SVPWM • load torque observer

\section{Introduction}

Induction motors have of late become the primary choice to drive industrial loads, especially where the applications require variable speed operations, easy to operate, physically robust, more efficient than DC motor and costefficient. The trend nowadays is to substitute DC machines with induction machines. Controlling the induction motor is not as easy as the DC motors because it is a multiple-input multiple-output (MIMO), nonlinear problem, and its operating conditions are away from the equilibrium points whereby linear approximation methods are not applicable (Riccardo and Cristiano, 2009; Vas, 1998). To get better performance of induction motor drives for variable speed drive applications various researches have been conducted by various scholars around the world. The control of AC machines can be classified in general in to two classes. The first and the oldest one is the scalar control. Even though this control scheme of $A C$ machines is easy to implement and can offer a better steady-state response, it is found to have a less robust dynamical response, and its response for parameter variation is awkward. Further research to achieve better variable speed performance on those machines has led to another control scheme called the Vector control. Vector control is a collective name given to field-oriented control (FOC), direct torque control (DTC), nonlinear control and predictive control of machines (Haitham Abu-Rub and Atif Iqbal, 2012).

An overview of researches being conducted on the control of induction motors shows that mostly the focus is on improving the DTC scheme of these motors. This is because of their superior performance over FOC scheme. To improve the conventional DTC, which was utilising hysteresis controllers as in Tibor et al. (2019), various research studies were proposed by several researchers. In Saber et al. (2017), the hysteresis controllers are replaced by proportional-integral $(\mathrm{PI})$ controllers to generate the reference voltages. Even though this controller improves the 
conventional direct torque controllers considering ripples, the application of these controllers needs an exact model of the system, which is ideal for practical considerations. Besides, selecting the controller gains for this controller is not an easy task, and the PI controllers are highly sensitive to disturbances, model uncertainties and parameter variations. In papers by Fu and Li (2015), Mesloub et al. (2016) and Uddin and Hafeez (2012), fuzzy logic, artificial neural network and model predictive control techniques are presented. The disadvantage of these techniques is their complex nature in solving tough and complex problems.

As a solution to the above-mentioned, the researchers started to focus on the nonlinear control of induction motors, especially the input-output feedback linearization (IOFL), which was preferred for its ultimate performance on robustness to varying parameters, simpler structure and quick reference tracking functionality (Lazreg and Bentaallah, 2018). IOFL is a technique that permits the designer/user to apply linear control strategies for nonlinear systems such as the induction motor (IM). This linearization method transforms the nonlinear system model into a linear equivalent one so that the linear control strategy can be utilised. This paper (Lascu et al., 2016) presents a design methodology in applying IOFL with PI flux controllers. However, this PI controller is still seen as a shortcoming for this technique. The solution to this problem is found by opting to use the robust type of controller family called the sliding mode controller (SMC). But, for fast tracking and better robustness, fractional order sliding mode controller (FOSMC) has opted in the speed controlling loop. Fractional counterparts of conventional controllers are nowadays a major concern for various scholars. The works of Tang et al. (2013) helped in the implementation of the antilock braking system, and Huang et al. (2012) detailed the way the position control of permanent magnet synchronous motor (PMSM) is applied and signifies how FOSMCs are effective over conventional integer order SMC.

In this paper, we have discussed nonlinear control of induction motor based on input-output feedback linearizing technique utilising space vector pulse width modulator (SVPWM). The advantage of this technique is obtained by compensating the nonlinearities present in the machine, and therefore, ensuring perfect decoupling between flux and electromagnetic torque. We utilised a model of the motor in $(\alpha \beta)$ frame. This model does not require further conversion to the direct and quadrature axis as this conversion will result in a delay for the controller to be designed. The major disadvantage of this controller scheme is that it requires all the states. As it is a challenging task to measure rotor flux and load torque disturbance, we have also designed a sliding mode flux observer from stator current and voltage information and PI type load observer. Another aspect that we are going to study in this paper is the speed controller. Speed has been controlled using conventional controllers such as PID, which is the most popular controller in electrical drives. However, such controllers could not guarantee the variations present in the system. The better option could be the FOSMC, which is a well-known controller that robustly controls systems with uncertainties. The application of FOSMC not only deals with robustness but also tracks the desired trajectory faster. The major drawback of using SMC families is the chattering (undesirable oscillations) present. This problem is alleviated by employing smooth continuous approximation of signum function.

\section{Mathematical model of induction motor in the stationary frame}

In control system engineering, before going to details of the controller designing step, the most important stage is in understanding the system and representing its dynamics nearly similar to as if it would be in the actual case. For our induction motor system, we used a mathematical model as it is found in Zaidi et al. (2014).

$$
\left\{\begin{array}{l}
\frac{d i_{s \alpha}}{d t}=-\gamma i_{s \alpha}+\alpha \beta \lambda_{r \alpha}+\beta \omega_{m} \lambda_{r \beta}+\frac{V_{s \alpha}}{\sigma} \\
\frac{d i_{s \beta}}{d t}=-\gamma i_{s \beta}-\beta \omega_{m} \lambda_{r \alpha}+\alpha \beta \lambda_{r \beta}+\frac{V_{s \beta}}{\sigma} \\
\frac{d \lambda_{r \alpha}}{d t}=\alpha L_{m} i_{s \alpha}-\alpha \lambda_{r \alpha}-\omega_{m} \lambda_{r \beta} \\
\frac{d \lambda_{r \beta}}{d t}=\alpha L_{m} i_{s \beta}+\omega_{m} \lambda_{r \alpha}-\alpha \lambda_{r \beta} \\
\frac{d \omega_{m}}{d t}=\mu\left(\lambda_{r \alpha} i_{s \beta}-\lambda_{r \beta} i_{s \alpha}\right)-k_{l} T_{l}-k_{f} \omega_{m}
\end{array}\right.
$$

Where variable notations are summarised as in Table 1. 
Table 1. Assignment for the variables

\begin{tabular}{llcl}
\hline Variable & Stands for & Variable & Stands for \\
\hline \hline$\mu$ & $p^{2} L_{m} / J_{e q} L_{r}$ & $\beta$ & $L_{m} / \sigma L_{r}$ \\
$\alpha$ & $R_{r} / L_{r}$ & $\gamma$ & $R_{s} / \sigma+\alpha \beta L_{m}$ \\
$\sigma$ & $L_{s}\left(1-L_{m}^{2} / L_{s} L_{r}\right)$ & $\rho$ & $\mu / k_{l}$ \\
$k_{l}$ & $\rho / J_{e q}$ & $k_{f}$ & $f_{r} / J_{e q}$ \\
\hline
\end{tabular}

\section{Design of space vector pulse width modulation}

$\mathrm{AC}$ motor control is mostly done by controlling the output voltage of DC/AC inverter, which in turn is fed to the stator of the machine by generating an appropriate switching gate pulse driving each power electronics switch. The first step is to determine the reference voltage and to find the sector where it is found. A step by step design procedure of SVPWM is presented in Panchal et al. (2013) for induction motor in $(d, q)$ reference frame.

$$
\overrightarrow{V_{s}}=V_{s \alpha}+j V_{s \beta},\left|\vec{V}_{r e f}\right|=\sqrt{V_{s \alpha}^{2}+V_{s \beta}^{2}}, \quad \theta=\tan ^{-1}\left(\frac{V_{s \beta}}{V_{s \alpha}}\right)
$$

The sector $S$ is decided by the value of $\theta$ and is categorised in one of the six regions illustrated in Figure 1. Application times

$$
\left\{\begin{array}{l}
T_{1}=\frac{\sqrt{3} T_{s}\left|\vec{V}_{r e f}\right|}{V_{d c}}\left(\sin \left(\frac{\pi}{3}-\theta+\frac{(S-1) \pi}{3}\right)\right) \\
T_{2}=\frac{\sqrt{3} T_{s}\left|\vec{V}_{r e f}\right|}{V_{d c}}\left(\sin \left(\theta-\frac{(S-1) \pi}{3}\right)\right) \\
T_{0}=T_{s}-\left(T_{1}+T_{2}\right)
\end{array}\right.
$$

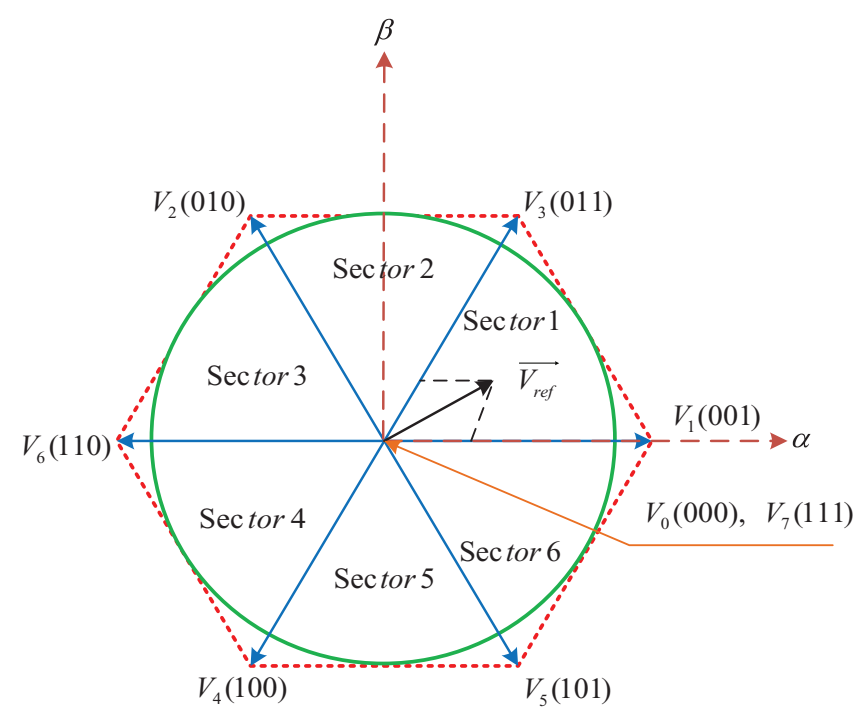

Fig. 1. Voltage space vector locations corresponding to different switching states 
After Dwell times or application times are determined, the next task is to calculate the duty cycle that is going to be modulated with a ramp signal of frequency equal to the frequency of the power electronic switching device. Ideal phase voltages outputs of the inverter can be computed from the DC input of the inverter and its basic gate pulses as follows:

$$
\left\{\begin{array}{l}
V_{a}=\frac{V_{d c}}{3}\left(2 p_{a}-p_{b}-p_{c}\right) \\
V_{b}=\frac{V_{d c}}{3}\left(2 p_{b}-p_{a}-p_{c}\right) \\
V_{c}=\frac{V_{d c}}{3}\left(2 p_{c}-p_{b}-p_{a}\right)
\end{array}\right.
$$

\section{Design of IOFL and FOSMC for speed controlling}

Nonlinear controllers are nowadays gaining popularity as digital technology going far away. Here in this paper, we applied nonlinear IOFL to exactly decouple electromagnetic torque and rotor flux. We also incorporate FOSMC from its integer counterpart for fast reference tracking. The general block diagram representation of the proposed system is presented in Figure 2.

\subsection{Design of IOFL}

For a nonlinear system, the following is a generalised form (Marquez, 2003; Slotine and Jean-Jacques, 1991):

$$
\left\{\begin{array}{l}
\dot{x}=f(x)+g(x) u \\
y=h(x)
\end{array}\right.
$$

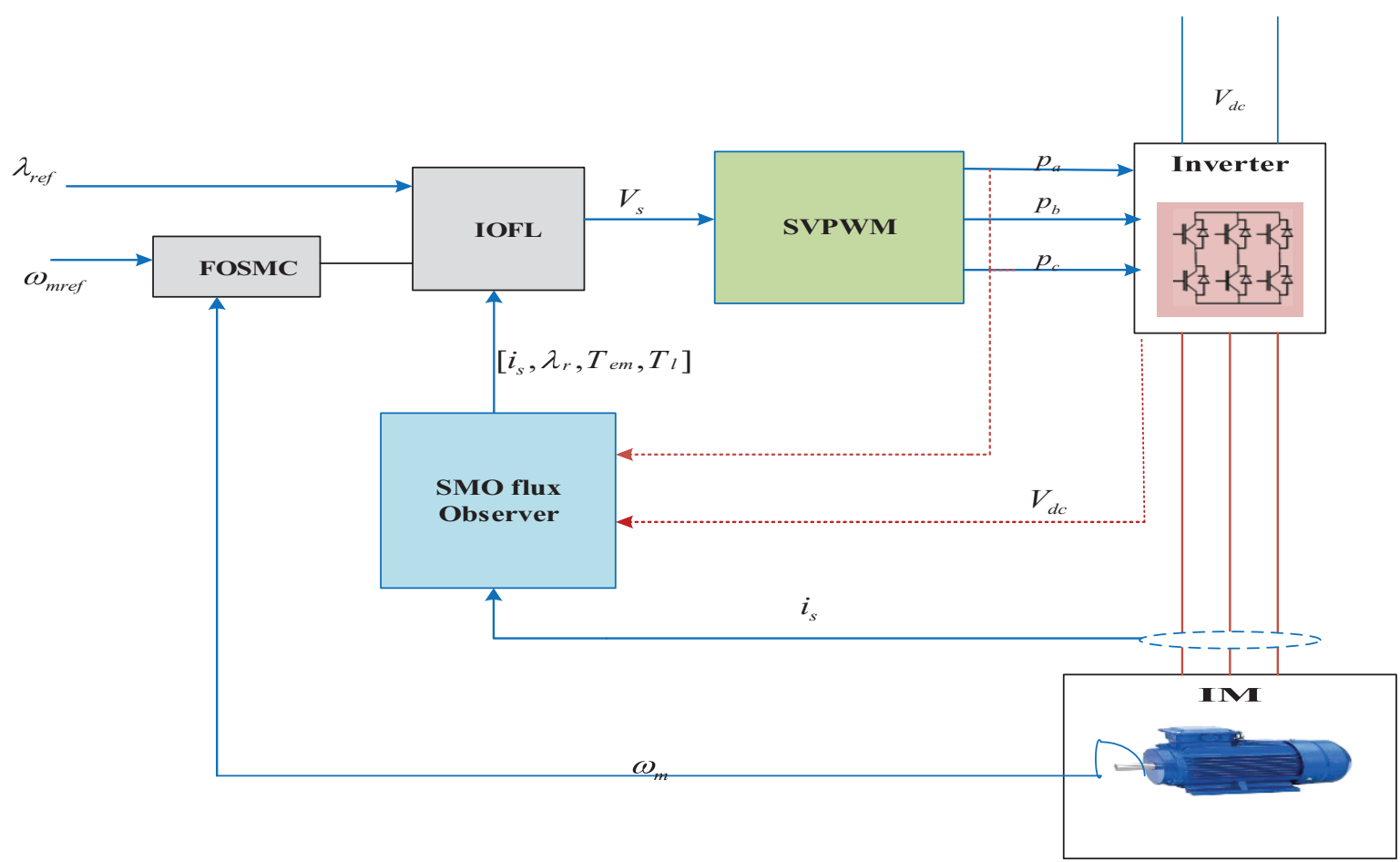

Fig. 2. Block diagram of FOSMC of feedback linearized induction motor 
To linearize a nonlinear system, one may use the input-state linearization technique. But, this linearization technique will not always result in a linearized output, and hence the IOFL is applied to get a linearized output equation too.

Taking successive differentiation of the output equation above leads to the following:

$$
\begin{aligned}
& \dot{y}=\frac{d(h)}{d t}[f(x)+g(x) u]=L_{f} h(x) \\
& \ddot{y}=\frac{d\left(L_{f} h\right)}{d t}[f(x)+g(x) u]=L_{f}^{2} h(x)+L_{g} L_{f} h(x)
\end{aligned}
$$

Where the following are the generalised context.

$$
y^{r}=L_{f}^{r} h(x)+L_{g} L_{f}^{r-1} h(x) u
$$

If $u$ does not appear in the equations of $y, y, \ldots, y^{r-1}$ and appears in the equation of $y^{r}$ with a non-zero coefficient, the system is input-output feedback linearizable and the state feedback control is

$$
u=\frac{1}{L_{g} L_{f}^{r-1} h(x)}\left[v-L_{f}^{r} h(x)\right]
$$

where $L_{f}^{k} h(x)=L_{f} L_{f}^{k-1} h(x)=\frac{\partial\left(L_{f}^{k-1} h\right)}{\partial x} f(x), v=y^{r}$.

In this case $r$, the maximum number of derivations until inputs appear is called the relative degree of the system. To ensure perfect tracking and to get the required behaviour, the auxiliary inputs can be defined as

$$
V_{i}=y_{i r e f}^{r_{i}}-k_{1} e-k_{2} e^{\prime}-\ldots-k_{r} e^{r-1}, \quad \text { Where } \quad e=y_{i}-y_{\text {iref }}
$$

In Eq. (10), $k_{r}$ is a positive coefficient that should be chosen to guarantee the system convergence.

The input-output linearization control is based on the model of the asynchronous machine in the $(\alpha, \beta)$ reference frame linked to the stator.

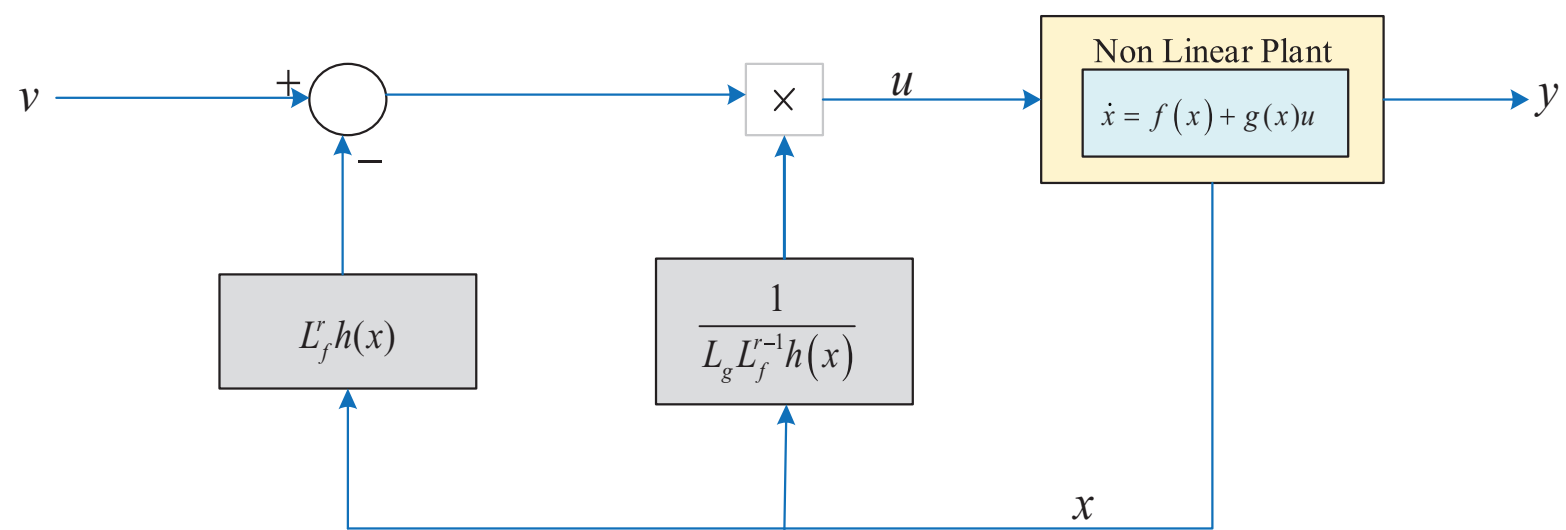

Fig. 3. General block diagram of IOFL 
Applying the above linearization concepts to our induction motor model presented in the modelling section of Eq. (1)

$$
\begin{aligned}
& f(x)=\left[\begin{array}{l}
-\gamma i_{s \alpha}+\alpha \beta \lambda_{r \alpha}+\beta \omega_{m} \lambda_{r \beta} \\
-\gamma i_{s \beta}-\beta \omega_{m} \lambda_{r \alpha}+\alpha \beta \lambda_{r \beta} \\
\alpha L_{m} i_{s \alpha}-\alpha \lambda_{r \alpha}-\omega_{m} \lambda_{r \beta} \\
\alpha L_{m} i_{s \beta}+\omega_{m} \lambda_{r \alpha}-\alpha \lambda_{r \beta} \\
\mu\left(\lambda_{r \alpha} i_{s \beta}-\lambda_{r \beta} i_{s \alpha}\right)-k_{l} T_{l}-k_{f} \omega_{m}
\end{array}\right], \quad g(x)=\left[\begin{array}{lllll}
\frac{1}{\sigma} & 0 & 0 & 0 & 0 \\
0 & \frac{1}{\sigma} & 0 & 0 & 0
\end{array}\right] \\
& x=\left[\begin{array}{lllll}
i_{s \alpha} & i_{s \beta} & \lambda_{r \alpha} & \lambda_{r \beta} & \omega_{m}
\end{array}\right]^{T}, \quad u=\left[\begin{array}{ll}
V_{s \alpha} & V_{s \beta}
\end{array}\right]^{T}, \quad h(x)=\left[\begin{array}{c}
y_{1} \\
y_{2}
\end{array}\right]=\left[\begin{array}{c}
T_{e m} \\
\lambda_{r \alpha}^{2}+\lambda_{r \beta}^{2}
\end{array}\right]
\end{aligned}
$$

Here, the outputs are considered as the motor's developed electromagnetic torque and the square of rotor flux. This can simplify the further derivation of the controller. Looking from the developed model, the system is considered as MIMO as it has two inputs namely $V_{s \alpha}$ and $V_{s \beta}$ and two outputs, namely, $y_{1}$ and $y_{2}$

Successive differentiation of the output until at least one of the inputs appear in the result gives us the following:

$$
T_{e m}=\rho\left(\lambda_{r \alpha} i_{s \beta}-\lambda_{r \beta} i_{s \alpha}\right)
$$

The derivative of electromagnetic torque is calculated as

$$
\begin{aligned}
\dot{y}_{1} & =L_{f} h_{1}(x)=\rho(\alpha+\gamma)\left(i_{s \alpha} \lambda_{s \beta}-i_{s \beta} \lambda_{s \alpha}\right)-\rho \omega_{m}\left(i_{s \alpha} \lambda_{s \alpha}+i_{s \beta} \lambda_{s \beta}\right) \\
& -\rho \beta \omega_{m}\left(\lambda_{r \alpha}^{2}+\lambda_{r \beta}^{2}\right)-\frac{\rho}{\sigma} \lambda_{r \beta} V_{s \alpha}+\frac{\rho}{\sigma} \lambda_{r \alpha} V_{s \beta}
\end{aligned}
$$

Performing a similar procedure for the second output, inputs appear in the second derivation

$$
\dot{y}_{2}=L_{f} h_{2}(x)=2 \lambda_{r \alpha} \dot{\lambda}_{r \alpha}+2 \lambda_{r \beta} \dot{\lambda}_{r \beta}
$$

Since one of the outputs does not appear in the result we proceed to the second derivative as

$$
\begin{aligned}
\ddot{y}_{2} & =L_{f}^{2} h_{2}(x)=-2 \alpha L_{m}(\gamma+3 \alpha)\left(i_{s \alpha} \lambda_{r \alpha}+i_{s \beta} \lambda_{r \beta}\right)-2 \alpha L_{m} \omega_{m}\left(i_{s \alpha} \lambda_{r \beta}-i_{s \beta} \lambda_{r \alpha}\right) \\
& +2 \alpha^{2}\left(2+\beta L_{m}\right)\left(\lambda_{r \alpha}^{2}+\lambda_{r \beta}^{2}\right)+2\left(\alpha L_{m}\right)^{2}\left(i_{s \alpha}^{2}+i_{s \beta}^{2}\right)+\frac{2 \alpha L_{m}}{\sigma} \lambda_{r \alpha} V_{s \alpha}+\frac{2 \alpha L_{m}}{\sigma} \lambda_{r \beta} V_{s \beta}
\end{aligned}
$$

Finally, the control law for IOFL of IM is designed as

$$
\left\{\begin{array}{l}
\dot{y}_{1}=v_{\alpha}=-k_{a 1}\left(T_{e m}-T_{e m r e f}\right)+T_{e m r e f}^{\prime} \\
\ddot{y}_{2}=v_{\beta}=-k_{b 1}\left(\lambda_{r}^{2}-\lambda_{r e f}^{2}\right)-k_{b 2}\left(\dot{\lambda}_{r}^{2}-\dot{\lambda}_{r e f}^{2}\right)+\ddot{\lambda}_{r e f}^{2}
\end{array}\right.
$$

The dynamics above will be stable if the roots of polynomials lie on the left side of the complex plane. This requires for the characteristic equation involving constants $k_{a 1}, k_{b 1}$ and $k_{b 2}$ to satisfy the Hutwith criterion.

\subsection{Proportional, integral and derivative speed controller}

Earlier we designed a nonlinear controller to independently control electromagnetic torque and flux based on the external references using IOFL. Flux reference is set by the user but torque reference is obtained from the speed controller block. Speed has been controlled using the PI controller, which is the most popular controller in electrical drives. But, the type of speed controller used matters. 
The governing differential equation for PID controller is defined as

$$
\frac{d \omega_{m}}{d t}=\frac{1}{J_{e q}}\left(T_{e m}-T_{l}\right)-\frac{f_{r}}{J_{e q}} \omega_{m}
$$

The gains of PID controllers should be tuned using an appropriate tuning mechanism. In this paper, we tuned it using the root locus tuning method as it is discussed in Bill and Dawn (2017).

\subsection{Design FOSMC for speed loop}

From the model equations derived earlier, the dynamics of the speed loop can be rearranged for simplifying our design approach without changing the dynamical structure.

Equation (17) can be rewritten as

$$
\begin{aligned}
\dot{\omega} & =\phi \omega_{m}+\ell\left(T_{e m}-T_{l}\right) \\
& =\left(\phi_{o}+\Delta \phi\right) \omega_{m}+\left(\ell_{o}+\Delta \ell\right)\left(T_{e m}-T_{l}\right) \\
& =\phi_{o} \omega_{m}+\ell_{o}\left(T_{e m}-T_{l}\right)+d
\end{aligned}
$$

where $\phi=-\frac{f_{r}}{J_{e q}}=\phi_{o}+\Delta \phi, \ell=\frac{1}{J_{e q}}=\ell_{o}+\Delta \ell, d=\Delta \phi \omega_{m}+\Delta \ell\left(T_{e m}-T_{l}\right)$.

' $\triangle$ ' Presents the parameter uncertainty and the subscript ' $O$ ' is used to indicate the nominal value of the parameter and ' $d$ ' is assigned for lumped uncertainties. To design the control law we defined the following sliding surface as Tabatabaei and Heidarpoor (2017) and followed the design approach sliding mode control as Grzegorz (2017) but here with fractional-order sliding surface.

$$
\begin{aligned}
& S_{\omega m}=e_{\omega m}+\lambda_{10} D_{t}^{-\alpha} e_{\omega m} \\
& \text { where } e_{\omega m}=\omega_{\text {mref }}-\omega_{m} \text { and } 0<\alpha<1
\end{aligned}
$$

Taking the time derivatives of the above-mentioned mathematical representations

$$
\begin{aligned}
\dot{S}_{\omega m} & =\dot{e}_{\omega m}+\lambda_{10} D_{t}^{1-\alpha} e_{\omega} \\
& =\left(\dot{\omega}_{m r e f}-\left(\phi_{o} \omega_{m}+\ell_{o}\left(T_{e m}-T_{l}\right)+d\right)\right)+\lambda_{10} D_{t}^{1-\alpha} e_{\omega}
\end{aligned}
$$

Now using the exponential reaching law

$$
\begin{aligned}
& \dot{S}_{\omega}=-k_{r} S_{\omega}-K_{s} \operatorname{sign}\left(S_{\omega}\right) \\
& T_{\text {emref }}=\frac{1}{\ell_{o}}\left[\dot{\omega}_{\text {mref }}-\phi_{o} \omega_{m}+\ell_{o} T_{l}+\lambda_{10} D_{t}^{1-\alpha} e_{\omega}+k_{r} S_{\omega}+K_{s} \operatorname{sign}\left(S_{\omega}\right)\right]
\end{aligned}
$$

The chattering present in the control input of this controller makes its implementation problematic as it can cause damages in the shaft and connected loads. Here, the improvement of this controller can be obtained by replacing the signum function with a smoother sigmoid function.

\subsection{Sliding mode rotor flux observer}

It is challenging to measure the rotor fluxes and feed them back to the controller. Therefore, we are obliged to design an observer for rotor flux states. Using our model equation presented in Eq. (1) and the techniques followed in Ben Regaya et al (2017), we can design a sliding mode estimator as follows. 
We can rewrite our previous model as

$$
\begin{aligned}
& \frac{d \overrightarrow{\hat{i}}_{s}}{d t}=\beta U-\frac{R_{s}}{\sigma} \overrightarrow{\hat{i}}_{s}+\frac{1}{\sigma} \vec{V}_{s} \\
& \frac{d \overrightarrow{\hat{\lambda}}_{r}}{d t}=-U
\end{aligned}
$$

where $U=\left[\begin{array}{cc}\alpha & \omega_{m} \\ -\omega_{m} & \alpha\end{array}\right]\left[\begin{array}{l}\lambda_{r \alpha} \\ \lambda_{r \beta}\end{array}\right]-\alpha L_{m}\left[\begin{array}{c}i_{s \alpha} \\ i_{s \beta}\end{array}\right]$ and terms with arrowhead represents a vector in $(\alpha, \beta)$ components.

A sliding mode observer can be designed by assuming the function $U$ is defined by the following matrix:

$$
U=\left[\begin{array}{c}
U_{s \alpha} \\
U_{s \beta}
\end{array}\right]=\left[\begin{array}{c}
-k_{s 1} \operatorname{sign}\left(S_{i 1}\right) \\
-k_{s 2} \operatorname{sign}\left(S_{i 2}\right)
\end{array}\right], \quad \text { where } \begin{gathered}
S_{i 1}=\hat{i}_{s \alpha}-i_{s \alpha} \\
S_{i 2}=\hat{i}_{s} \beta-i_{s \beta}
\end{gathered}
$$

The observer expression is, therefore

$$
\begin{aligned}
& \frac{d \overrightarrow{\hat{i}}_{s}}{d t}=\beta U-\frac{R_{s}}{\sigma} \overrightarrow{\hat{i}}_{s}+\frac{1}{\sigma} \vec{V}_{s} \\
& \frac{d \overrightarrow{\hat{\lambda}}_{r}}{d t}=-U
\end{aligned}
$$

\subsection{Load torque observer}

In the SMCs mentioned above, one can notice that the $T_{\text {l }}$ term is present in the control law expression. But, this mechanical shaft load disturbance is an unknown term or it is difficult to determine its value using measurement techniques. This necessitates the formulation of a load torque observer that can extract its value from estimates of flux and current values. In this paper, the method used to get the load torque estimate of PMSM in Benchabane et al (2010) is applied (Figure 4).

$$
\widehat{T}_{l}=k_{p 1}\left(\widehat{\omega_{m}}-\omega_{m}\right)+k_{i 1} \int\left(\widehat{\omega_{m}}-\omega_{m}\right) d t
$$

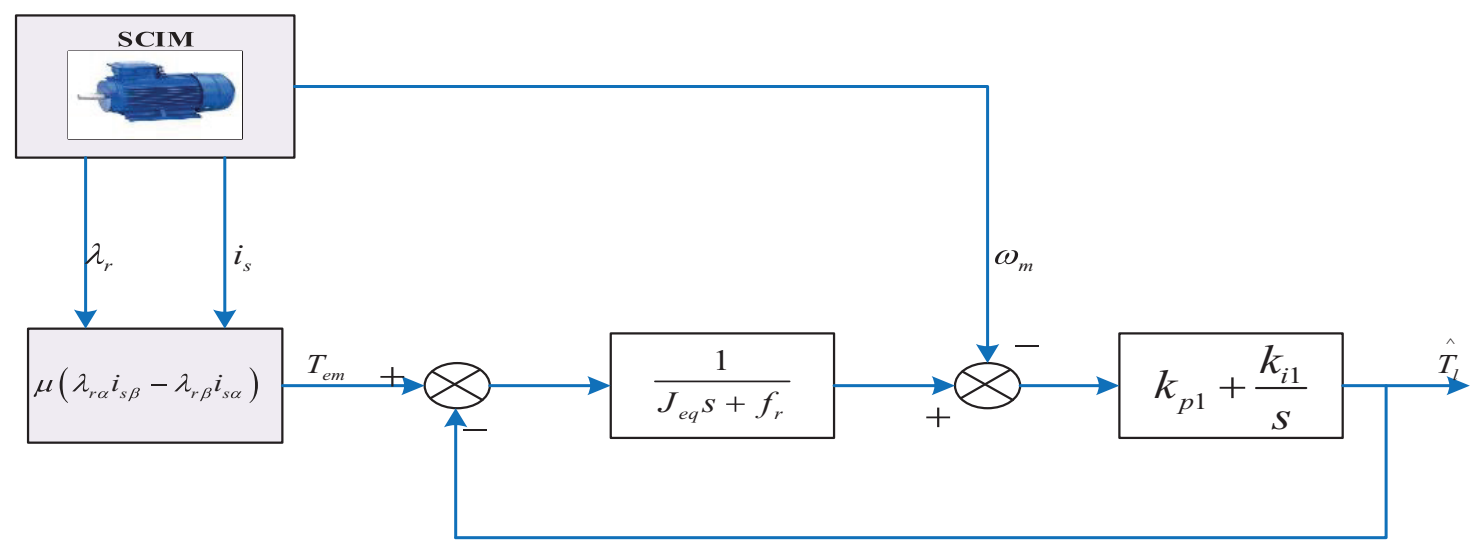

Fig. 4. Load torque observer 
Where

$$
\widehat{\omega_{m}}=\frac{1}{j_{e q} s+f_{r}}\left(T_{e}-\widehat{T}_{l}\right)
$$

\section{Simulation results and discussions}

\subsection{Simulation with proposed controller and observers}

In this section, simulation results with the proposed controller are presented. Simulation results are given for the tests: first starting with a speed set point of $120 \mathrm{rad} / \mathrm{s}$, starting with a flux set point of $3 \mathrm{~Wb}$ and second by applying a load torque ( $\mathrm{TI}$ ) profile indicated later, and then responses of the proposed system for varying speed and flux reference is presented.

\subsubsection{Responses with no application of load disturbance}

Figures 5-7 show no-load responses of rotor speed, phase stator currents and the rotor flux trajectory obtained from PID and the proposed FOSMC, respectively. First, Figure 5 presents a comparative study

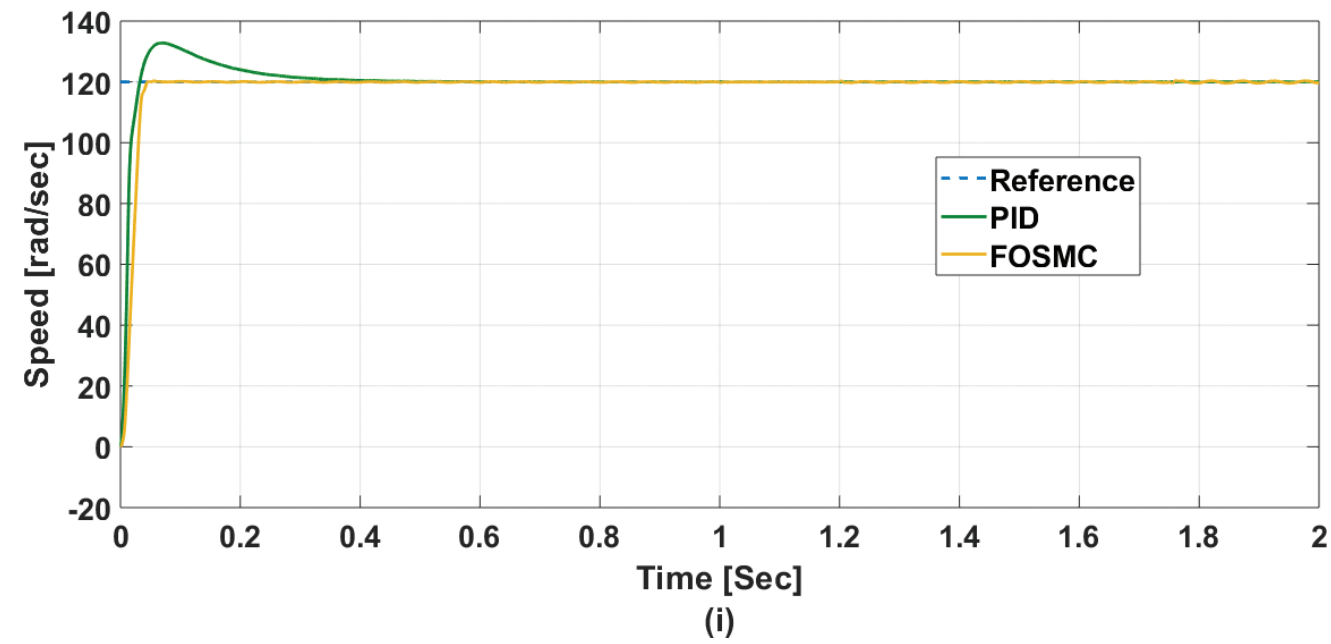

Fig. 5. Speed responses with no-load condition

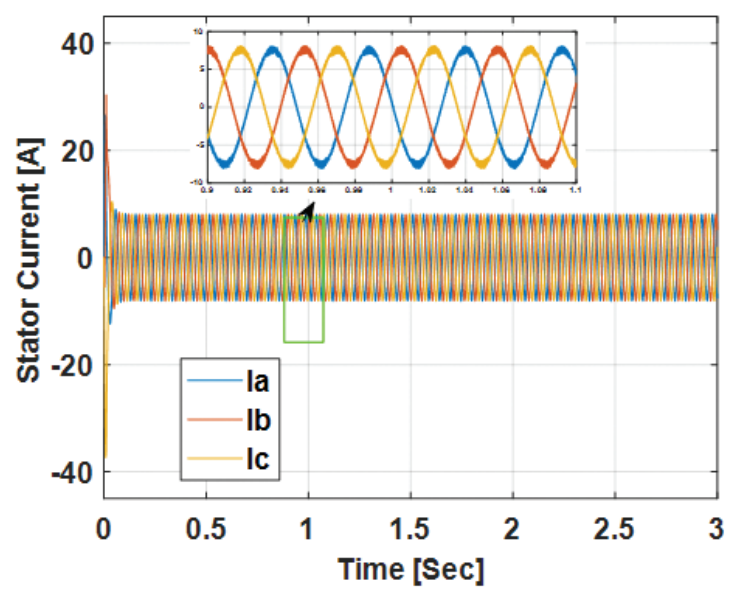

(i)

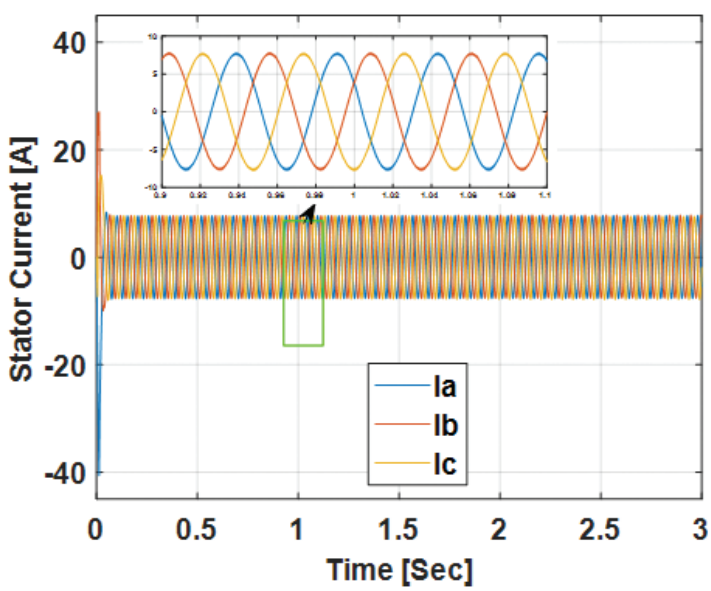

(ii)

Fig. 6. Stator current responses at no-load condition 


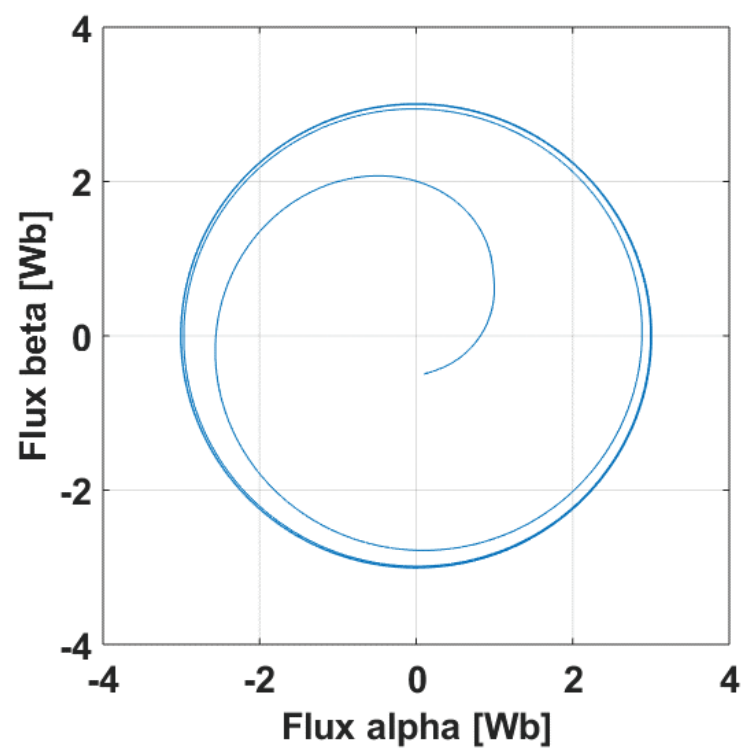

(i)

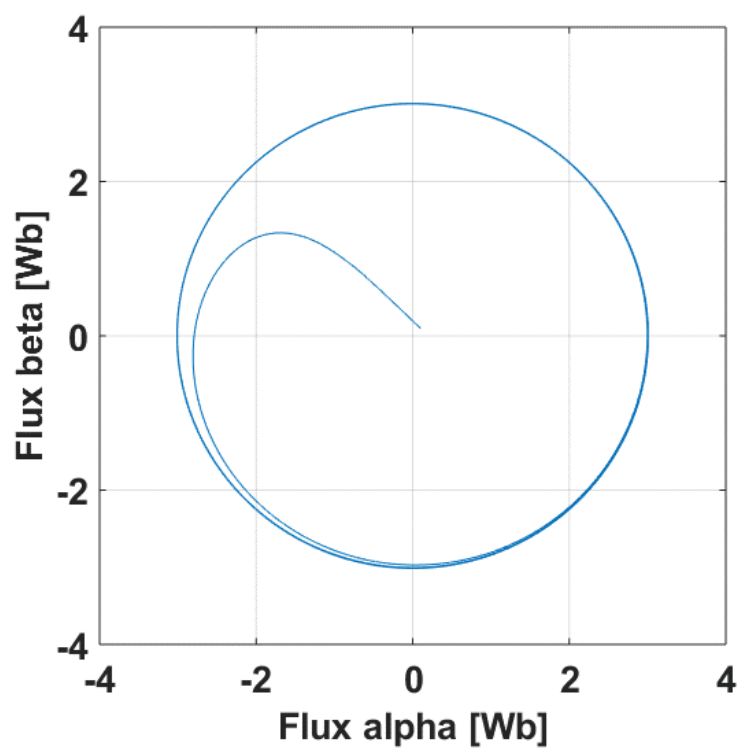

(ii)

Fig. 7. Rotor flux trajectory

in terms of speed response with $120 \mathrm{rad} / \mathrm{s}$ as a reference speed with $3 \mathrm{~Wb}$ flux magnitude reference. Referring to this figure, it is shown that both techniques offer a good static and dynamic response when starting up. It can be noticed that IOFL based on FOSMC has faster speed response and shorter settling time. From the response plots of Figure 6(i) for PID and Figure 6(ii) for FOSMC, it can be observed that good sinusoid waveform for stator current are obtained by applying FOSMC and looking at Figures 7 (i) and 7(ii) for PID and FOSMC, respectively, better rotor flux circular trajectory is noticed for FOSMC.

\subsubsection{Simulation with applied load disturbance}

As can be noticed from the first plot of Figure 8, speed droppings are higher in PID, while FOSMC has a more robust response to this load disturbance. The second plot in this figure presents electromagnetic torque responses due to the application of load with a profile in the plot of Figure 9 (left). Observation from this plot informs us that FOSMC leads to minimisation of torque ripples. Figure 9 (left) also shows applied load torque and estimated torque using the proposed load estimator. Here the best estimate of load disturbance is obtained. This indicates better performance of the applied load estimation using an implemented estimator. The final plot in Figure 9 (right end) presents reference, actual and estimated rotor flux modulus. This plot indicates the implemented sliding mode rotor flux observer that leads to a good estimate of rotor flux.

\subsubsection{Simulation for varying speed and flux references}

The tracking performance of the IM is observed under the varying speed reference command in Figure 10 (left) and the varying flux magnitude reference command is shown in Figure 10 (right). Simulation plots reveal that the actual speed tracking of commonly used PID speed controller is characterised by its excessive overshoot during instantaneous changes of speed and flux, but FOSMC shows almost no overshoots. Besides, speed tracking can be inferred from a zoomed view. In Figure 11, even though the $(\alpha, \beta)$ sinusoidal waves of rotor flux seem almost similar to the two-speed controllers, a closer look of trajectories in (iii) and (iv) reveals a well recognisable circular path for FOSMC. 

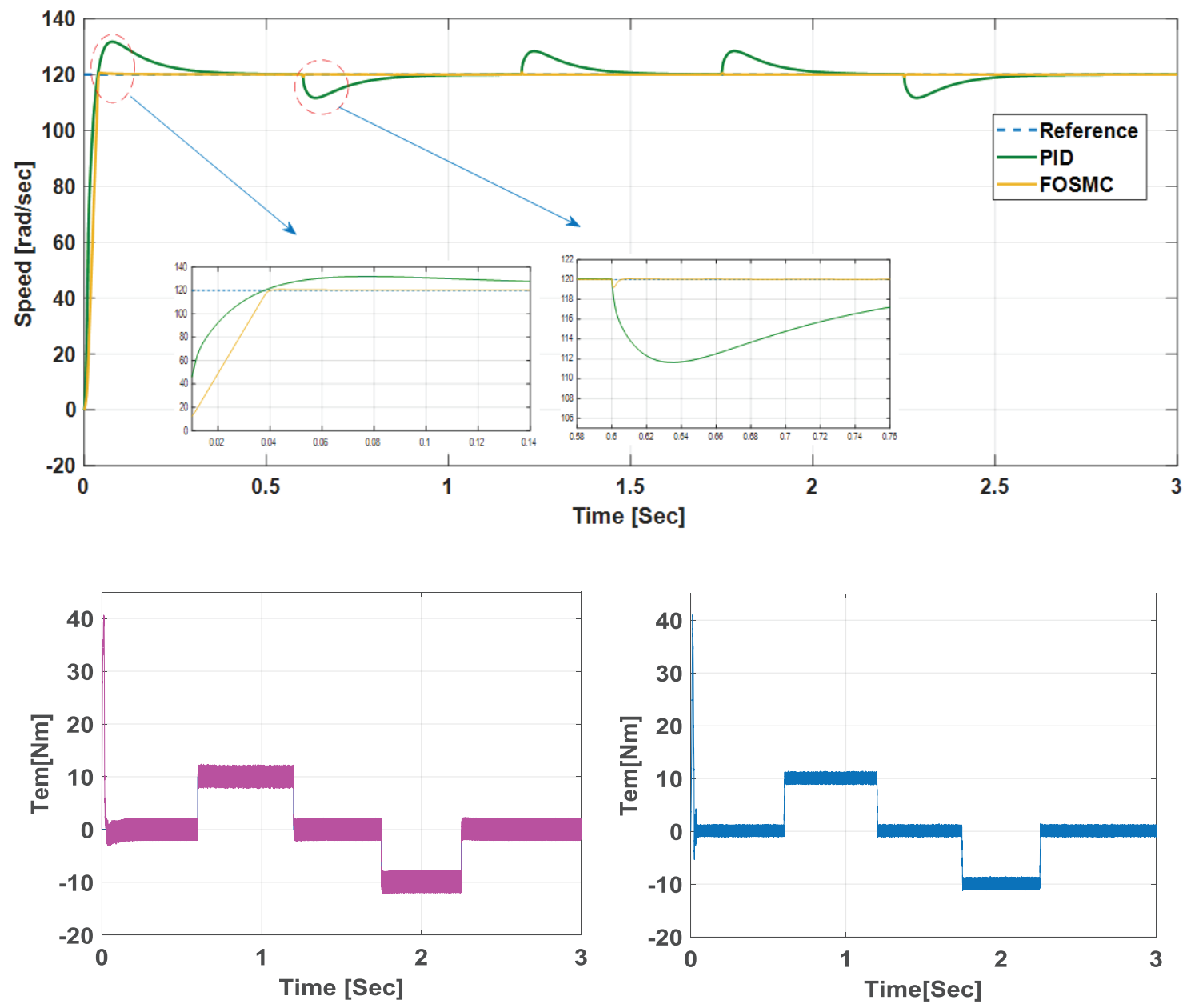

Fig. 8. Developed electromagnetic torque
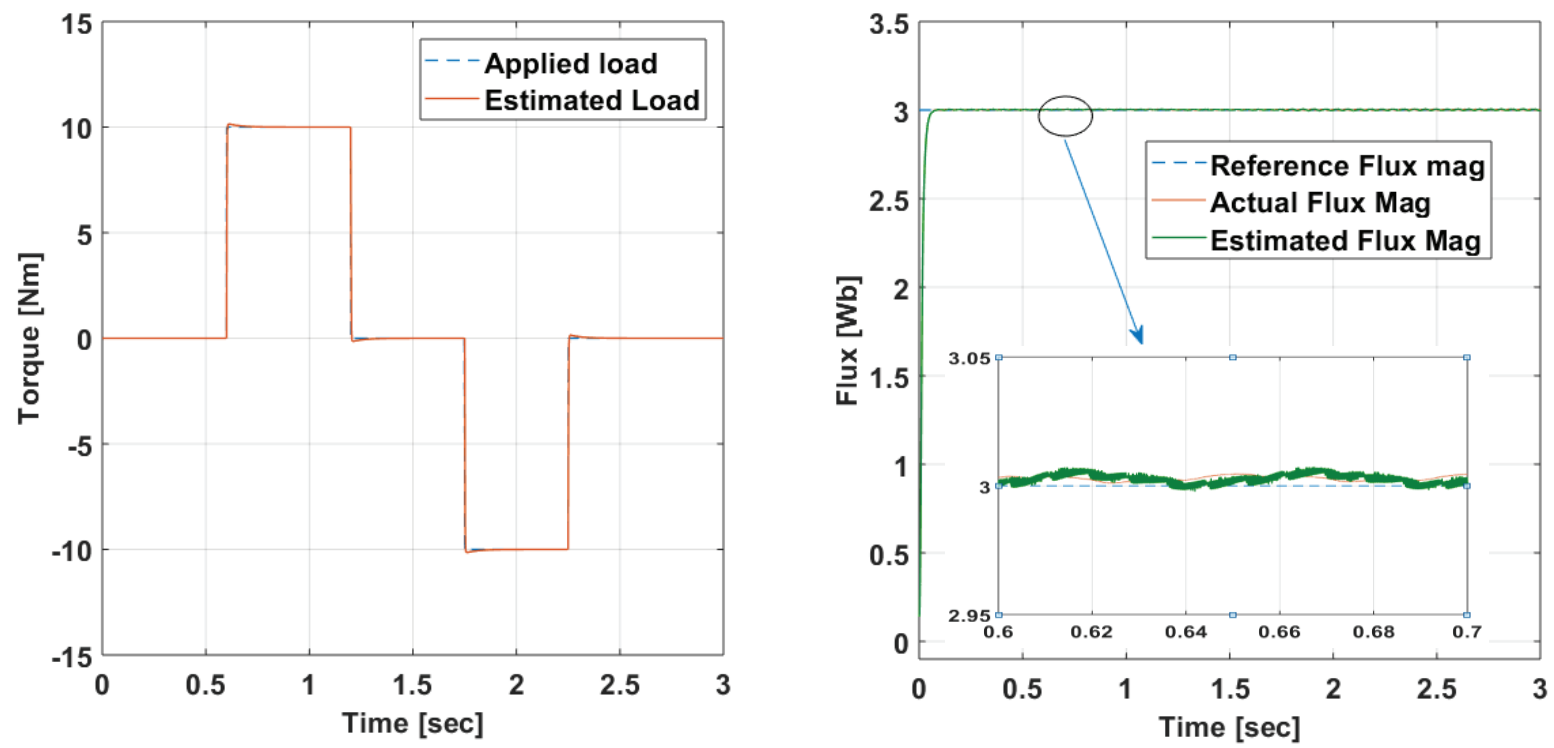

Fig. 9. Applied and estimated load using PID and FOSMC as speed controller 

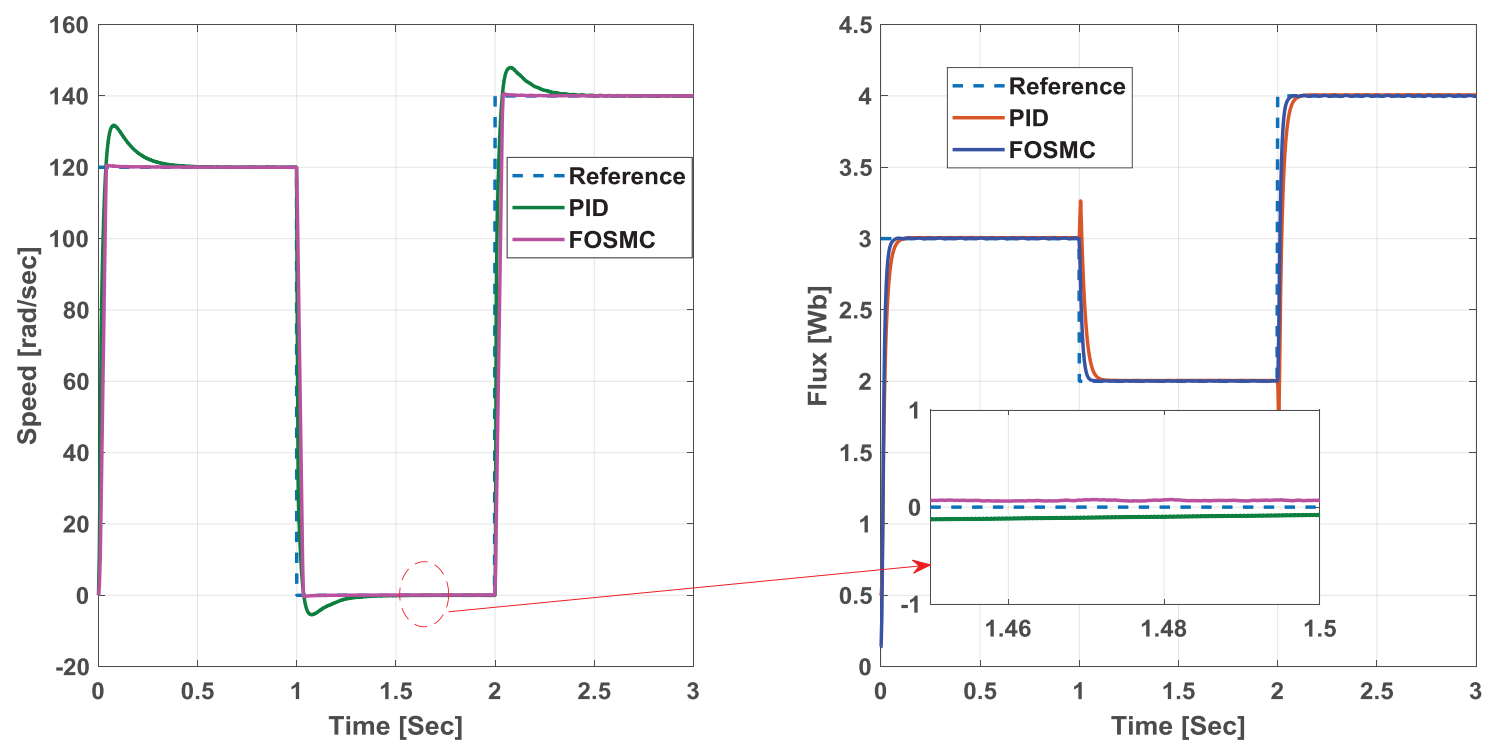

Fig. 10. Response for a varying speed (left) and varying flux in (right)

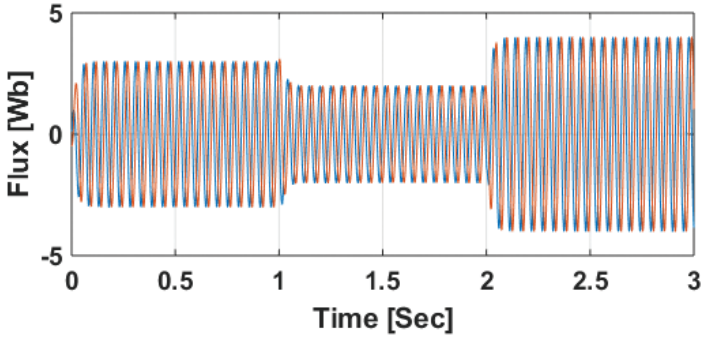

(i)

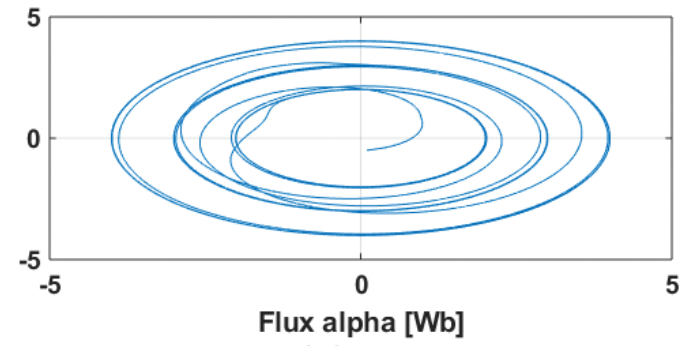

(iii)

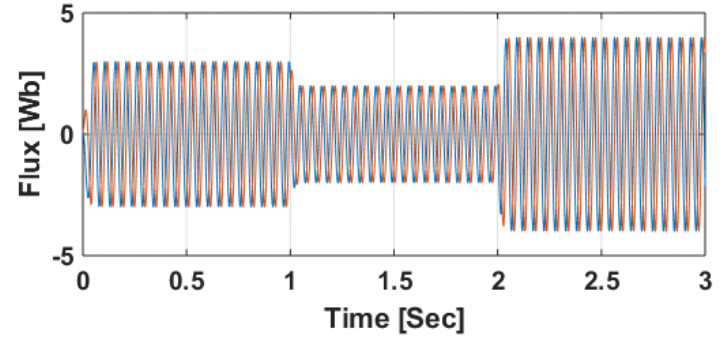

(ii)

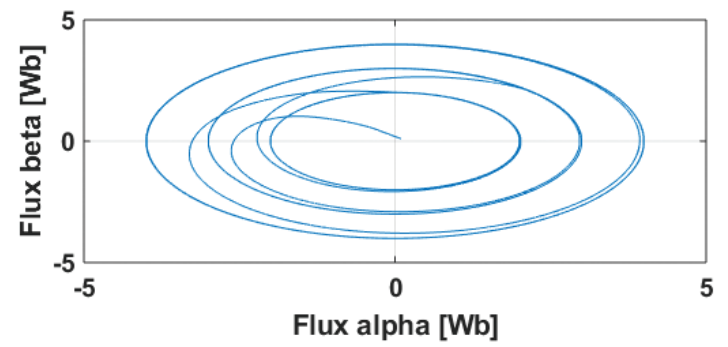

(iv)

Fig. 11. Rotor flux sinusoidal waveform and its trajectory

\section{Conclusion}

In this paper, the performance of IOFL of squirrel cage induction motor with two controllers namely, PID and fractional-order speed controller based on sliding mode rotor-flux estimator is presented. The rotor flux components are obtained from SM observer using measured stator current and stator voltages calculated from inverter basic switching pulses and measured DC input of the inverter. A load disturbance estimator with a PI type controller extracts the applied disturbance well. The stability of the proposed FOSMC algorithm has been analysed mathematically using the Lyapunov stability theory. Simulation results show a perfect decoupling between the two subsystems (flux and torque), and it also shows good dynamic behaviour and the control performances. The designed FOSMC has increased the robustness of the control approach against reference 
speed variation and external load disturbance. Moreover, this controller is found to have interesting tracking performance of varying reference flux, which is impossible in FOC schemes as it depends on the assumption of constant flux reference.

\section{References}

Ben Regaya, C., Farhani, F. and Zaafouri, A. (2017). An Adaptive Sliding-Mode Speed Observer. ICIC Express Letters, 11(4), pp. 763-771.

Benchabane, F., Titaouine, A., Taibi, D. and Yahia, K. (2010). Systematic fuzzy sliding mode approach combined with extended Kalman filter for permanent magnet synchronous motor control. In: Proceedings of the IEEE International Conference on Systems, Man and Cybernetics, 10-13 October 2010, Istanbul, Turkey, No. 7, pp. 2169-2174.

Bill, M. and Dawn, T. (2017). Control Tutorials for MATLAB and Simulink - Motor Position Root Locus Controller Design, [online]. Available at: http://ctms.engin.umich.edu/CTMS/index.php?exa $\mathrm{mple}=$ MotorPosition\&section=ControlRootLocus. [Accessed 15 Nov. 2019].

Fu, X. and Li, S. (2015). A Novel Neural Network Vector Control Technique for Induction Motor Drive. IEEE Transactions on Energy Conversion, 30, pp. 1428-1437.

Grzegorz, T. (2017). Sliding Mode Speed Control of an Induction Motor Drive Using Time-Varying Switching Line*. Power Electronics Drives, 2(37), pp. 105-120.

Haitham Abu-Rub, J. G. and Iqbal, A. (2012). High Performance Control of AC Drives with Matlab/ Simulink Models High Performance Control of AC Drives with Matlab/Simulink. Chichester, West Sussex: John Wiley \& Sons, Ltd., Publication.

Huang, J., Li, H., Chen, Y. and Xu, Q. (2012). Robust Position Control of PMSM Using Fractional-Order Sliding Mode Controller. Abstract and Applied Analysis, 2012, pp. 1-33.

Lascu, C., Jafarzadeh, S., Fadali, S. M. and Frede, B. (2016). Direct Torque Control with Feedback Linearization for Induction Motor Drives. IEEE Transactions on Power Electronics, 1, pp. 1-9.

Lazreg, M. H. and Bentaallah, A. (2018). Input Output Linearization Control of Double Star Induction Machine. Revue Roumaine des Sciences Techniques - Serie Électrotechnique et Énergétique, 63(6), pp. 423-428.

Marquez, H. J. (2003). Nonlinear Control Systems Analysis and Design. New Jersey: John Wiley \& Sons, Inc., Publication.
Mesloub, H., Benchouia, M. and Goléa, A. (2016). Predictive DTC Schemes with PI Regulator and Particle Swarm Optimization for PMSM Drive: Comparative Simulation and Experimental Study. The International Journal of Advanced Manufacturing Technology, 86, pp. 3123-3134.

Panchal, S. N., Sheth, V. S. and Pandya, A. A. (2013). Simulation Analysis of SVPWM Inverter Fed Induction Motor Drives. Ashoka Technologies, 2, pp. 18-22.

Riccardo, M. V. and Cristiano, P. T. (2009). Advances in Industrial Control. London: Springer-Verlag London Limited 2010.

Saber, K., Soufien, G., Abdellatif, M. and Mimouni, M. F. (2017). Implementation on the FPGA of DTC-SVM Based Proportional Integral and Sliding Mode Controllers of an Induction Motor: A Comparative Study. Journal of Circuits, Systems, and Computers, 26(3), pp. 1-32.

Slotine, L. and Jean-Jacques, E. (1991). Applied Nonlinear Control. New Jersey: Prentice Hall.

Tabatabaei, M. and Heidarpoor, S. (2017). Speed Control of a DC Motor Using a Fractional Order Sliding Mode Controller. In: 2017 IEEE Industrial and Commercial Power Systems Europe (EEEIC/ I\&CPS Europe), 6-9 June 2017, Milan, Italy, No. 3, pp. 1057-1060.

Tang, Y., Zhang, X., Zhang, D., Zhao, G. and Guan, X. (2013). Fractional Order Sliding Mode Controller Design for Antilock Braking Systems. Neurocomputing, 111, pp. 122-130.

Tibor, V., László, S. and Handler, Á. (2019). An Investigation of Direct Torque Control and Hysteresis Current Vector Control for Motion Control Synchronous Reluctance Motor Applications. Power Electronics and Drives, 4(39), pp. 115-124.

Uddin, M. and Hafeez, M. (2012). FLC-Based DTC Scheme to Improve the Dynamic Performance of an IM Drive. IEEE Transactions on Industry Applications, 48(2), pp. 823-831.

Vas, P. (1998). Sensorless Vector and Direct Torque Control. New York: Oxford University Press.

Zaidi, S., Naceri, F. and Abdssamed, R. (2014). InputOutput Linearization of an Induction Motor Using MRAS Observer. International Journal of Advanced Science and Technology, 68, pp. 49-56. 


\section{Appendix}

\section{A.1. List of variable designations}

$\mathrm{IM}$, induction motor

$V_{d c}$, DC voltage fed to the inverter

$V_{s a}, V_{s \beta}, i_{s a^{\prime}}, i_{s \beta}$, stator voltage and currents in $(\alpha \beta)$ plane

$\lambda_{r \alpha}, \lambda_{r \beta}$, rotor fluxes in $(\alpha \beta)$ plane

$R_{s}, R_{r}$, stator and rotor resistances

$L_{s}, L_{r}$, stator and rotor inductances

$L_{m}$, mutual inductance

$J_{e q}, f_{r}$, moment of inertia and coefficient of friction

$T_{e m}, T_{l}$, electromagnetic torque and applied torque

$p$, number of pole pairs

\section{A.2. Value of motor parameters}

Table A1. Squirrel cage induction motor parameters

\begin{tabular}{|c|c|c|c|}
\hline \multicolumn{4}{|c|}{ Motor parameters } \\
\hline \multicolumn{2}{|l|}{ Specifications } & \multicolumn{2}{|c|}{ Parameters } \\
\hline Rated power & $1.5 \mathrm{~kW}$ & $R_{s}$ & $4.6 \mathrm{~W}$ \\
\hline Rated Voltage & $400 \mathrm{~V}$ & $R_{r}$ & $4.35 \mathrm{H}$ \\
\hline Rated Current & $3.45 \mathrm{~A}$ & $L_{s}, L_{r}$ & $0.3382 \mathrm{H}$ \\
\hline Rated Frequency & $50 \mathrm{~Hz}$ & $L_{m}$ & $0.3210 \mathrm{H}$ \\
\hline Number of pole pairs & 2 & $J_{e q}$ & $0.004 \mathrm{Kg} \times \mathrm{m}^{-2}$ \\
\hline Rated speed & $1400 \mathrm{rpm}$ & $f_{r}$ & $0.0 .0010 \mathrm{Nm} \times \mathrm{s} \times \mathrm{rad}^{-1}$ \\
\hline \multicolumn{4}{|c|}{ Controller and estimator gains } \\
\hline \multicolumn{2}{|c|}{ Controller or estimator block } & \multicolumn{2}{|c|}{ Involved gains } \\
\hline \multicolumn{2}{|l|}{ IOFL } & \multicolumn{2}{|c|}{$k_{a 1}=8 \times 10^{3}, k_{b 1}=4 \times 10^{5} \cdot k_{b 2}=8 \times 10^{3}$} \\
\hline \multicolumn{2}{|l|}{ FOSMC and PID } & \multicolumn{2}{|c|}{$\lambda_{1}=1.5, \alpha=0.2, K_{r}=4, K_{s}=8 \times 10^{3}, k_{p}=0.924, k_{i}=8.40, k_{d}=0.0084$} \\
\hline \multicolumn{2}{|c|}{ Flux estimator and load estimator } & \multicolumn{2}{|c|}{$k_{s 1}=k_{s 2}=4 \times 10^{3}, k_{p 1}=3.9, k_{i 1}=66.4$} \\
\hline
\end{tabular}

FOSMC, fractional order sliding mode controller; IOFL, input-output feedback linearization. 